\title{
A Time to Event Analysis of Adverse Drug Reactions Due to Tenofovir, Zidovudine and Stavudine in a Cohort of Patients Receiving Antiretroviral Treatment at an Outpatient Clinic in Zimbabwe
}

\author{
Tinashe Mudzviti1,2*, Nyasha T. Mudzongo', Samuel Gavi3 ${ }^{3}$ Cleophas Chimbetete², \\ Charles C. Maponga ${ }^{1,4}$, Gene D. Morse ${ }^{4}$ \\ ${ }^{1}$ School of Pharmacy, University of Zimbabwe, Harare, Zimbabwe \\ ${ }^{2}$ Newlands Clinic, Harare, Zimbabwe \\ ${ }^{3}$ Department of Clinical Pharmacology, College of Health Sciences, Harare, Zimbabwe \\ ${ }^{4}$ Center of Excellence in Bioinformatics and Life Sciences and The School of Pharmacy and Pharmaceutical \\ Sciences, University at Buffalo, SUNY, Buffalo, NY, USA \\ Email: ${ }^{*}$ tmudzviti@yahoo.co.uk
}

Received 5 December 2014; accepted 19 March 2015; published 24 March 2015

Copyright (C) 2015 by authors and Scientific Research Publishing Inc.

This work is licensed under the Creative Commons Attribution International License (CC BY).

http://creativecommons.org/licenses/by/4.0/

cc) (7)

Open Access

\section{Abstract}

Background: Achieving the long terms goals of antiretroviral treatment (ART) requires a careful approach during treatment initiation that takes into account patient's psychosocial state, availability and accessibility of treatment combinations, and adherence support. Adverse drug reactions that occur during the initial phases have a bearing on treatment outcomes and thus need to be monitored and treated. Objective: This study was done to assess length of time (survival time) it took for clinically significant adverse drug reactions to occur in patients taking Nucleoside Reverse Transcriptase Inhibitors (N(t)RTI) available for treatment of Human Immunodeficiency Virus (HIV) infection in Zimbabwe. Methods: A retrospective cohort of patient data collected from January 2009 to December 2012 was extracted from an Electronic Health Record database. Data from patients who were initiated on antiretroviral (ARV) drug regimens containing $N(t) R T I$ drugs were analysed for survival time. A sample of 205 patient files was extracted for the time period for survival analysis using adverse drug reactions due to N(t)RTI drugs. Results: After data extraction, a total of 205 patient records were used in determining the time to event analysis of ADR's in the cohort. The age range for the patients included in the study was $9-76$ with a mean of 41 years (s.d = 14.8). Patients initiated on stavudine had a lower survival time before a clinically significant

How to cite this paper: Mudzviti, T., Mudzongo, N.T., Gavi, S., Chimbetete, C., Maponga, C.C. and Morse, G.D. (2015) A Time to Event Analysis of Adverse Drug Reactions Due to Tenofovir, Zidovudine and Stavudine in a Cohort of Patients Receiving Antiretroviral Treatment at an Outpatient Clinic in Zimbabwe. Pharmacology \& Pharmacy, 6, 201-206. 
ADR compared to tenofovir $(-365$ days, $p$-value $<0.0005)$. Patients on zidovudine also had a less time before a significant reaction compared to those on tenofovir $(-230$ days; $p$-value $=0.008)$. Patients on zidovudine fared better compared to those on stavudine $(-134$ days; $p$-value $<0.0005)$. The mean survival time was highest for tenofovir (618 days), followed by zidovudine ( 388 days), and then stavudine (254 days).Conclusion: Patients on tenofovir have a longer survival time before a clinically significant adverse reaction. Treatment programmes need to continue commencing patients on tenofovir containing regimens as patients can be maintained for longer periods on this regimen.

\section{Keywords}

\section{Survival Analysis, Electronic Medical Records, Adverse Drug Reactions}

\section{Introduction}

The introduction of new antiretroviral (ARV) drug classes continues to improve the available options for patients on treatment making it easier for care providers to tailor treatments to a single patient. These developments have made it easier to achieve the goals of ARV treatment in many patients and thus improving overall survival and quality of life. Addition of new drug combinations and regimens is complemented by the patient's ability to adhere to prescribed treatment in the long term. Adherence has been identified as one of the most important factors in ensuring robust treatment outcomes in patients. Adherence to antiretroviral treatment has also been shown to be dependent on a number of factors like pill burden, existence of psychosocial support structure, the patient's readiness to start treatment, age [1]; and possibly the most important factor of all the type and severity of adverse drug reactions experienced by the patient [2] [3].

Ideally a patient on ARV treatment should be maintained on the first and initial treatment regimen for as long as possible. In many cases patients may change treatment combinations within a year of initiating therapy because of adverse drug reactions that warrant treatment change [4]. Virologic suppression by various nucleoside reverse transcriptase inhibitors (NRTI) containing drug regimens has been shown to be equal including rates of drug switching, however differences occurred in adverse drug reaction profiles of the NRTI drugs [4]. In resource limited countries the choice on NRTI drugs to initiate or maintain patients on is limited with most countries resorting to the use of stavudine (D4T), zidovudine (AZT) or tenofovir (TDF) as part of NRTI backbone.

So far only a few studies set in resource-poor-settings and using routine program data have compared TDF, d4T (30 mg), and AZT-based regimens maybe because data on TDF is limited particularly in sub-Saharan Africa where the drug has been in use for a short duration. In Zambia they compared single drug substitutions and composite mortality and loss-from-care endpoint and findings will be outlined below as with the other two. In Lesotho they assessed single drug substitutions and all-cause mortality. Bygrave and colleagues in South Africa using longer term data and including virologic response, and robust loss-from-care outcomes have compared on the following outcomes: single drug substitutions, HIV RNA suppression, CD4 count increase, loss-from care, and mortality [5].

Tenofovir containing drug regimens in some cases showed better treatment outcomes and a favourable safety profile compared to stavudine and zidovudine [5] [6]. The relative safety of tenofovir as shown in clinical studies and its ability for sustained immune recovery has seen a lot of changes in treatment guidelines, with tenofovir being the N(t)RTI of choice [7]. Tenofovir generally has lower substitution rates compared to zidovudine and stavudine containing regimens [5] [8]-[10]. In spite of the evidence that currently exist that points to differences in efficacy and safety of NRTI's in management of HIV infection, a lot of countries do not have options for HIV patients. Most HIV infected patients in developing countries have either been on stavudine or zidovudine containing regimens before the introduction of tenofovir. New World Health Organization (WHO) recommendations to use tenofovir in favour of zidovudine or stavudine have been slowly implemented across Zimbabwe. Due to the changes in treatment guidelines patients are continuously being upgraded to new regimens. The main objective of this study was to determine the time to clinically significant adverse drug reactions in a cohort of treatment naïve patients on different $\mathrm{N}(\mathrm{t}) \mathrm{RTI}$ containing ARV regimens. 


\section{Methods}

We carried out a retrospective analysis of a cohort of patients who were initiated on ARV treatment at Newlands Clinical in Harare, Zimbabwe. The clinic provides outpatient care and treatment to HIV infected patients from marginalized urban and peri-urban communities. Data were collected from patients who were initiated on ARV treatment from January 2009 to December 2012 and had been switched to another NRTI drug due to a clinically significant adverse drug reaction. The clinic uses an Electronic Medical Record Health System called ePOC (electronic point of care) enabling an integration of HIV and TB therapy. Time to event was considered to be the time a patient started taking treatment up to the time NRTI was substituted due to a clinically significant adverse reaction. Covariates that were collected included age, gender, level of education, employment status, date of HIV diagnosis, date of ARV treatment initiation. Statistical analysis was done using STATA Version 13, a total of 205 patient records were extracted for analysis during the period under review. Ethical approval for the study was provided by the Joint Research and Ethics Committee of the University of Zimbabwe (JREC/306/13).

\section{Results}

A study sample of 205 patient files is extracted from ePOC. The demographic characteristics of the study participants are shown in Table 1.

The age range for the patients included in the study was 9 - 76 with a mean of 41 years $($ s.d $=14.8)$. The majority of the participants were female and most participants were on a zidovudine containing regimen. The mean number of days before a clinically significant adverse drug reaction due to an N(t)RTI was lowest for stavudine (254 days), followed by zidovudine (388 days) and tenofovir (618 days).

Table 2 shows that patients on stavudine had a lower survival time before a clinically significant ADR compared to tenofovir ( -365 days, p-value $<0.0005)$. Patients on zidovudine also had a less time before a significant reaction compared to those on tenofovir $(-230$ days; $p$-value $=0.008)$. Patients on zidovudine fared better compared to those on stavudine $(-134$ days; $\mathrm{p}$-value $<0.0005)$.

Patients on tenofovir had a longer survival time before a clinically significant adverse drug reaction followed by those on zidovudine; patients on stavudine had the lowest survival time (Table 3).

Figure 1. Survival in the three groups of N(t)RTI’s (AZT vs. TDF vs. D4T).

\section{Discussion}

Our study showed that the type of NRTI agent used had an impact on the time to occurrence of an adverse effect.

Table 1. Demographic characteristics of study participants.
\begin{tabular}{cc}
\hline Characteristic & Frequency, n(\%) \\
\hline $9-<20$ & $26(12.7)$ \\
$20-<30$ & $17(8.3)$ \\
$30-<40$ & $44(21.5)$ \\
$40-<50$ & $64(31.2)$ \\
$50-<60$ & $34(16.6)$ \\
$\geq 60$ & $20(9.8)$ \\
Gender & \\
Male & $91(44.4)$ \\
Female & $114(55.6)$ \\
N(t)RTI & \\
Zidovudine & $116(56.6)$ \\
Stavudine & $69(33.7)$ \\
Tenofovir & $20(9.8)$ \\
\hline
\end{tabular}


Table 2. Linear regression-comparison of time before treatment change due to ADR by N(t)RTI.

\begin{tabular}{ccccc}
\hline NRTI & $\begin{array}{c}\text { Observed } \\
\text { Coefficient }\end{array}$ & $\begin{array}{c}\text { Bootstrap } \\
\text { Standard Error (5000 reps) }\end{array}$ & P-Value & 95\% Confidence Interval \\
\hline Tenofovir vs. Stavudine & -365 & 86.4 & $<0.0005$ & $-535 ;-196$ \\
Tenofovir vs. Zidovudine & -230 & 86.2 & 0.008 & $-399 ;-61$ \\
Zidovudine vs. Stavudine & -134 & 33.0 & $<0.0005$ & $-200 ;-70$ \\
\hline
\end{tabular}

Table 3. Mean survival time for patients initiating on AZT, D4T, and TDF.

\begin{tabular}{cccc}
\hline N(t)RTI & Restricted Mean (days) & Standard Error & 95\% Confidence Interval \\
\hline Tenofovir & 618 & 80.8 & $460.4-777.3$ \\
Zidovudine & 388 & 24.3 & $340.9-436.2$ \\
Stavudine & 254 & 22.3 & $210.0-297.3$ \\
\hline
\end{tabular}

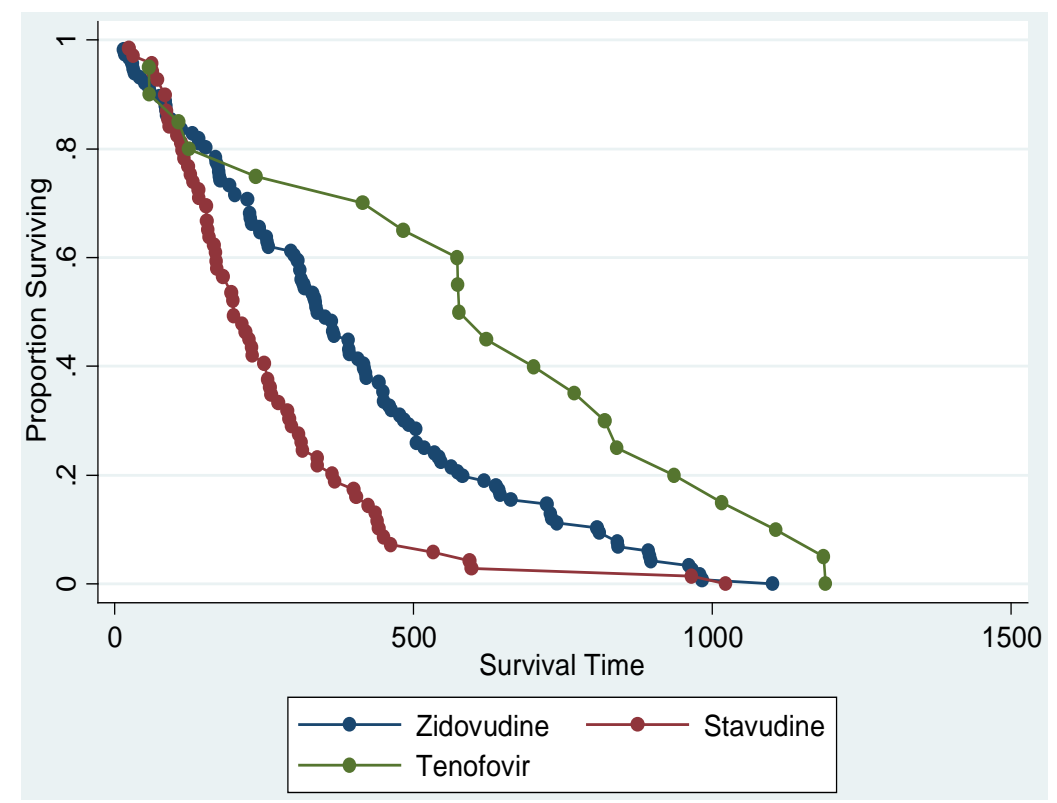

Figure 1. Shows significant difference in survival probability between patients initiated on the three N(t)RTI's; stavudine, zidovudine, and tenofovir. The likelihood-ratio test statistic of homogeneity by N(t)RTI gave a chi-square test statistic $\left\{\operatorname{chi}^{2}(2)=15.3\right.$, P-value $\left.=0.0005\right\}$.

The results showed that a greater proportion of patients maintained tenofovir therapy for longer periods compared to zidovudine and stavudine respectively. The mean number of days before a clinically significant adverse drug reaction due to an N(t)RTI was lowest for stavudine (254 days), followed by zidovudine (388 days) and tenofovir (618 days). Bygrave and colleagues have also reported on outcomes and toxicities among patients on TDF, zidovudine, and d4T-based first-line ART in a routine treatment cohort in Lesotho [5]. Before the 2010 ART recommendations, only a few countries in Africa (Zambia, Namibia, Lesotho, and Botswana) had adopted TDF as part of the first line treatment regimen. Thus the researchers of this study noted a scarcity of reporting of outcomes on TDF compared with other regimens in low-resource settings. All adult patients initiating antiretroviral therapy from January 1, 2008, to December 31, 2008, were included in the analysis and followed until December 31, 2009. They reported that patients on zidovudine were more than twice as likely to experience a toxicity-driven regimen substitution compared with tenofovir (adjusted hazard ratio: 2.32, 95\% confidence interval: 1.23 to 4.40); for patients on stavudine, the risk of a toxicity-driven regimen switch was almost 6 times higher than tenofovir (adjusted hazard ratio: 5.43, 95\% confidence interval: 3.31 to 8.9. They concluded that a tenofovir-based first-line regimen is supportive of simplified care by reducing the rate of regimen substitutions 
compared with stavudine-based and zidovudine-based regimens [5].

Njuguna et al. in their study described incidence rates of drug substitutions and regimen switches due to virological failure among patients receiving regimens containing D4T, AZT or TDF in a large peri-urban area in Gugulethu, Cape Town, South Africa. They also sought to characterize the reasons and risk factors for ADRrelated drug substitutions. This retrospective cohort study included HIV positive, ART naïve adults aged $>18$ years. Follow-up was censored at first drug change and analysis focused on NRTI changes only. Results indicated that virologic suppression rates at 1 year, regimen switching due to virologic failure and overall losses to the programme were similar across the three groups. TDF had the lowest incidence rate of drug substitutions (2.6 per 100 person-years) compared to 17.9 for D4T and 8.5 per 100 person-years for AZT. ADRs accounted for the majority of drug substitutions of D4T. They concluded that regimen switches and virologic suppression were similar for patients exposed to TDF, D4T and AZT, suggesting that all regimens were equally effective. However, TDF was found to be better tolerated with a substantially lower rate of drug substitutions due to ADRs [11].

In our study, the likelihood ratio test statistic of homogeneity yielded a chi square value of 15.28 and a p value of 0.00048053 which implies that the differences noted are significant; The lowess smoother curve demonstrated that stavudine had the most number of switches within the first 500 days of treatment. In the Kaplan Meir analyses tenofovir had the highest proportion of participants surviving at any given time followed by zidovudine and lastly stavudine. This supports what is already known in literature that a tenofovir based regimen performs better than AZT and D4T. An example of such a study which reported on this outcome is a study by Velen et al. [6]. Whilst Velen et al., looked at proportions of patients that developed adverse events as a result of an N(t)RTI, our study set to determine the duration that patients were maintained an a regimen until toxicities resulting in a switch emerged.

This study supports the continued expansion and roll out of tenofovir as the preferred first line N(t)RTI in the management of ART naïve patients. Just as well, when a patient eventually fails first-line ART, AZT is the NRTI of choice for patients initiating second line therapy. It is however important to note that in Zimbabwe the national guidelines still list D4T as a possible NRTI option for patients who have not been able to tolerate the other alternatives.

\section{Conclusion}

There was a statistically significant difference in the time to N(t)RTI switch as a result of adverse drug event. Patients on TDF tolerated this medicine for longer when compared to patients on AZT whilst D4T was the NRTI which was tolerated for the shortest duration.

\section{Acknowledgements}

The authors would like to thank Newlands Clinic for the support. This manuscript was supported by Award Number D43TW007991 from the Fogarty International Center. The content is solely the responsibility of the authors and does not necessarily represent the official views of the Fogarty International Center or the National Institutes of Health. For the remaining authors, there are no conflicts of interests to declare. Tinashe Mudzviti is a fellow of the Letten Foundation Research Centre.

\section{References}

[1] Langat, N.T., Odero, W. and Gatongi, P. (2012) Antiretroviral Drug Adherence by HIV Infected Children Attending Kericho District Hospital, Kenya. East African Journal of Public Health, 9, 101-104.

[2] Rajesh, R., Sudha, V., Varma, D. and Sonika, S. (2012) Association between Medication Adherence Outcomes and Adverse Drug Reactions to Highly Active Antiretroviral Therapy in Indian Human Immunodeficiency Virus-Positive Patients. Journal of Young Pharmacists, 4, 250-260. http://dx.doi.org/10.4103/0975-1483.104369

[3] Falang, K.D., Akubaka, P. and Jimam, N.S. (2012) Patient Factors Impacting Antiretroviral Drug Adherence in a Nigerian Tertiary Hospital. Journal of Pharmacology and Pharmacotherapeutics, 3, 138-142.

[4] Njuguna, C., Orrell, C., Kaplan, R., Bekker, L.G., Wood, R. and Lawn, S.D. (2013) Rates of Switching Antiretroviral Drugs in a Primary Care Service in South Africa before and after Introduction of Tenofovir. PLoS ONE, 8, e63596. http://dx.doi.org/10.1371/journal.pone.0063596

[5] Bygrave, H., Ford, N., van Cutsem, G., Hilderbrand, K., Jouquet, G., Goemaere, E., et al. (2011) Implementing a Te- 
nofovir-Based First-Line Regimen in Rural Lesotho: Clinical Outcomes and Toxicities after Two Years. Journal of Acquired Immune Deficiency Syndromes, 56, e75-e78. http://dx.doi.org/10.1097/QAI.0b013e3182097505

[6] Velen, K., Lewis, J.J., Charalambous, S., Grant, A.D., Churchyard, G.J. and Hoffmann, C.J. (2013) Comparison of Tenofovir, Zidovudine, or Stavudine as Part of First-Line Antiretroviral Therapy in a Resource-Limited-Setting: A Cohort Study. PLoS ONE, 8, e64459. http://dx.doi.org/10.1371/journal.pone.0064459

[7] Cassetti, I., Madruga, J.V., Suleiman, J.M., Etzel, A., Zhong, L., Cheng, A.K., et al. (2007) The Safety and Efficacy of Tenofovir DF in Combination with Lamivudine and Efavirenz through 6 Years in Antiretroviral-Naive HIV-1-Infected Patients. HIV Clinical Trials, 8, 164-172. http://dx.doi.org/10.1371/journal.pone.0064459

[8] Arribas, J.R., Pozniak, A.L., Gallant, J.E., Dejesus, E., Gazzard, B., Campo, R.E., et al. (2008) Tenofovir Disoproxil Fumarate, Emtricitabine, and Efavirenz Compared with Zidovudine/Lamivudine and Efavirenz in Treatment-Naive Patients: 144-Week Analysis. Journal of Acquired Immune Deficiency Syndromes, 47, 74-78. http://dx.doi.org/10.1097/QAI.0b013e31815acab8

[9] Chi, B.H., Mwango, A., Giganti, M., Mulenga, L.B., Tambatamba-Chapula, B., Reid, S.E., et al. (2010) Early Clinical and Programmatic Outcomes with Tenofovir-Based Antiretroviral Therapy in Zambia. Journal of Acquired Immune Deficiency Syndromes, 54, 63-70.

[10] Spaulding, A., Rutherford, G.W. and Siegfried, N. (2010) Tenofovir or Zidovudine in Three-Drug Combination Therapy with One Nucleoside Reverse Transcriptase Inhibitor and One Non-Nucleoside Reverse Transcriptase Inhibitor for Initial Treatment of HIV Infection in Antiretroviral-Naive Individuals. Cochrane Database of Systematic Reviews (CDSR), 10, Article ID: CD008740.

[11] Njuguna, C., Orrell, C., Kaplan, R., et al. (2013) Rates of Switching Antiretroviral Drugs in a Primary Care Service in South Africa before and after Introduction of Tenofovir. PLOS ONE, 8, e63596. http://dx.doi.org/10.1371/journal.pone.0063596 